\title{
Indicadores de identificación de riesgo temprano de alteración de la atención conjunta en el Trastorno del Espectro Autista
}

\section{Early risk identification indicators of alteration of joint attention in Autism Spectrum Disorder}

\author{
Claudia Ximena González-Moreno \\ Pontificia Universidad Javeriana, Colombia \\ (Rec: marzo de 2018 - Acept: octubre de 2018)
}

\begin{abstract}
Resumen
La cognición social comienza con el desarrollo infantil de la atención conjunta en la interacción comunicativa con los otros. El objetivo de este artículo es reflexionar sobre los indicadores de identificación de riesgo temprano de alteración de la atención conjunta en el Trastorno del Espectro Autista (TEA). De esta manera, se aporta información que posibilita determinar las estrategias de intervención que requiere un niño con TEA desde las etapas más tempranas de su vida. Esta información proporciona herramientas para orientar al contexto psicológico y educativo con niños con TEA. Se discute la importancia de considerar la atención conjunta como la base del desarrollo de la cognición social en una construcción interactiva y dialógica con los otros.
\end{abstract}

Palabras clave: Atención conjunta, Trastorno del Espectro Autista, cognición social, intencionalidad, habilidades pro-sociales.

\begin{abstract}
Social cognition begins with the child's development of joint attention in the communicative interaction with others. The objective of this article is to reflect on the Early risk identification indicators of alteration of joint attention in Autism Spectrum Disorder (ASD). Thus, information is provided to determine the intervention strategies that a child with ASD requires from the earliest stages of life. This information provides tools to guide the psychological and educational context of children with ASD. The article discusses the importance of considering joint attention as the basis for social cognition development in an interactive and dialogical construction with others.
\end{abstract}

Keywords: Joint attention, Autism Spectrum Disorder, social cognition, intentionality, pro-social skills.

Correspondencia a: Claudia Ximena Gozález-Moreno. Email: clauxigo@hotmail.com 


\section{Introducción}

El Trastorno del Espectro Autista (TEA) es una alteración del neurodesarrollo (Rivière, 1997, 2001a) que se expresa en dificultades en el desarrollo de habilidades pro-sociales características de la cognición social, por ejemplo, en la posibilidad de ponerse en el lugar del otro para responder emocionalmente ante las experiencias afectivas de otras personas en la interacción comunicativa (Baron-Cohen, 1988; Baron-Cohen et al, 1997; Rivière y Martos, 2000; Rivière, 2001; Barbaro \& Dissanayake, 2009; Zwaigenbaum, Bryson \& Garon, 2013). De igual manera, se advierten dificultades: para atribuir estados mentales en los otros -intenciones, deseos, pensamientos(García, 1995); para imitar gestos (Williams, Whiten \& Singh, 2004); para la comunicación, la relación social, la colaboración y la capacidad de compartir la perspectiva del otro; para representar mentalmente a otra persona, comprendiendo sus intenciones, creencias o emociones; para extraer inferencias causales sobre su comportamiento; y para percibir e interpretar las señales sociales que emanan de los ojos, la cara, la postura del cuerpo y la voz y, de esta manera, interpretar el significado del comportamiento o el lenguaje de los demás (Baron-Cohen, 1995; Scaife y Bruner, 1975; Tomasello et al., 2005; Wimmer y Perner, 1983; Mundy, 2017).

La comprensión del TEA se constituye en un desafío. Cuando el niño es pequeño puede ser difícil su identificación. Sin embargo, la detección precoz de alteraciones en la atención conjunta en niños que presentan TEA permite diseñar progra-

Figura 1. Flujograma PRISMA para la selección de publicaciones

Figura 1. Flujograma PRISMA para la selección de publicaciones mas interventivos que facilitan su desarrollo psicológico. Por esta razón, los objetivos de este artículo son: primero describir cómo se desarrolla la atención conjunta y establecer su relación con la cognición social en el TEA y, segundo, resaltar la importancia de la evaluación temprana en el TEA. Además, este trabajo pretende sugerir algunos indicadores de identificación temprana de alteración de la atención conjunta en el TEA y algunas formas de intervención que involucran a la familia.

\section{Método}

La búsqueda principal se llevó a cabo en las bases de datos de Scopus, PubMed, ISI Web of Sciences y ACM Digital Library. Los documentos fueron extraídos de las bases de datos usando los siguientes términos de búsqueda solos o en combinación utilizando el operador lógico "Y": "atención conjunta", "Trastorno del Espectro Autista", "cognición social". Posteriormente, se agregó un filtro por idioma, seleccionando inglés, español y portugués. Se incluyeron artículos publicados entre 1986 y 2018.

Se hizo una revisión narrativa, se analizó la relevancia de los títulos y los resúmenes de acuerdo con los objetivos y a continuación, se incluyeron los artículos seleccionados. También se analizaron algunos libros de referencia relacionados con los temas tratados. La estrategia de búsqueda se muestra en el diagrama de flujo PRISMA de la figura 1.

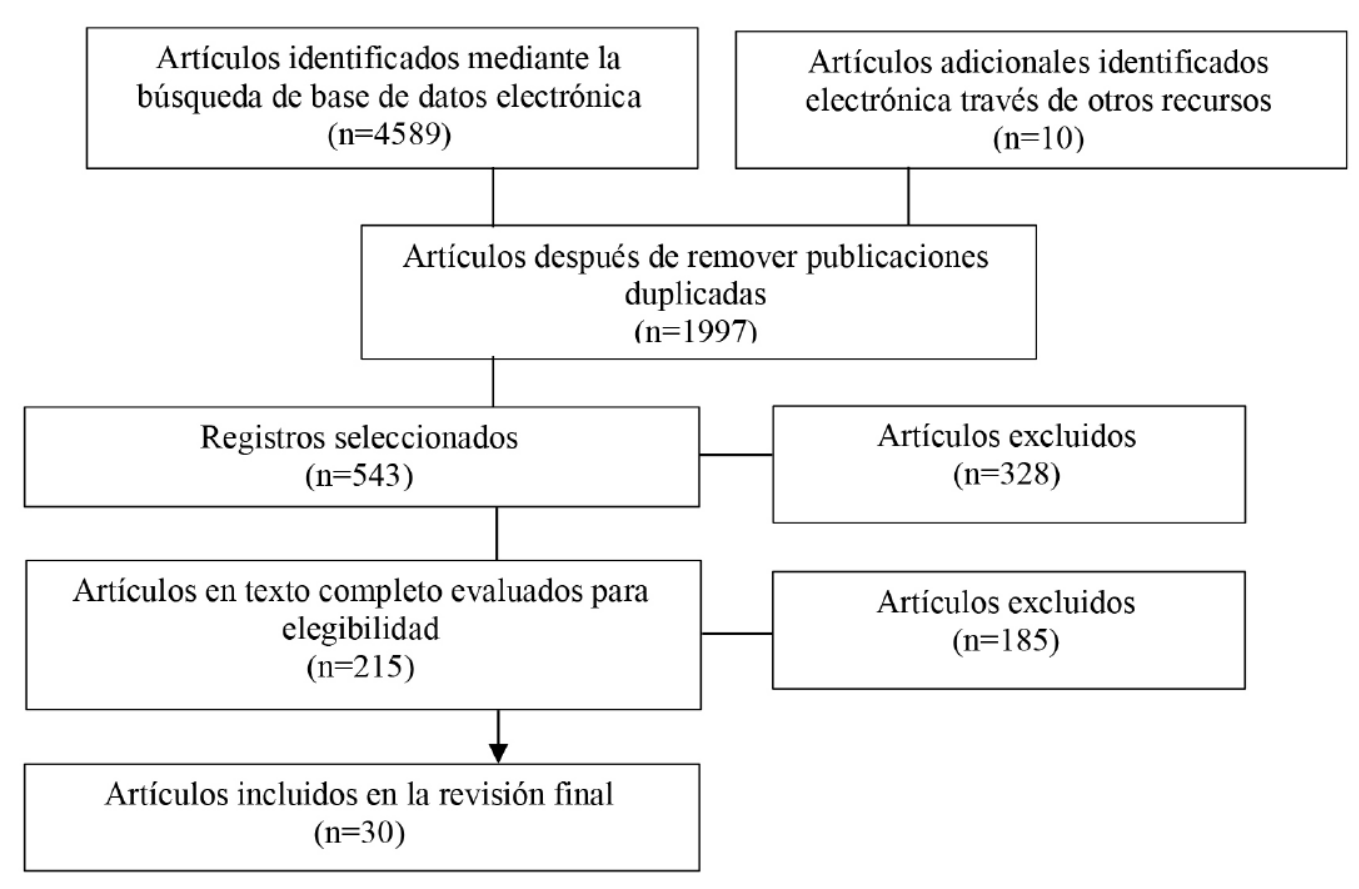


Como criterios de inclusión se planteó que las investigaciones fueran revisiones, estudios experimentales (analíticos) o cuasiexperimentales donde se considerara el desarrollo de la atención conjunta en niños con TEA, y que los artículos estuvieran publicados en revistas científicas. Frente a criterios de exclusión se planteó que no debían ser estudios de carácter psicométrico en donde solo se presentan datos numéricos. Para la selección final de los artículos se consideraron aquellos cuyo texto completo estuviera disponible para lectura o descarga.

Para analizar la producción científica en relación con la atención conjunta, se agruparon las investigaciones en nueve tópi-

Tabla 1. Resumen de investigaciones sobre atención conjunta, cognición social y autismo cos de relevancia en la literatura: 1) sobre la atención conjunta y su papel en el desarrollo de la cognición social, 2) sobre formas de interacción: diádicas y triádicas, 3) sobre la mente dialógica, 4) sobre la dedicación conjunta como habilidad de la comprobación de la atención, 5) sobre el seguimiento de la mirada, 6) sobre la adopción de perspectivas, 7) sobre atención conjunta y juego simbólico, 8) sobre evaluación en el TEA , 9) sobre intervención dirigida al desarrollo de la atención conjunta en el TEA.En la tabla 1 se presenta el resumen de algunos de los principales hallazgos de los artículos revisados.

\section{Fuente}

Loveland y Landry (1986)

Mundy y Sigman (1989)

Kasari, Sigman, Mundy y Yirmiya (1990)

Mundy, Kasari y Sigman (1992)

Baron-Cohen (1993)

Mundy et al. (1994)

Mundy (1995)

Mundy y Crowson (1997)

Mundy et al. (2000)

Tomasello (2007)

Leekam, López y Moore (2001)

Mundy y Neal (2001)

Kasari, Freeman \& Paparella (2006)

Mundy y Thorp (2005)

Leekam (2005)

Mundy (2018)

González-Moreno (2018a)

\section{Breve resumen}

Los niños con TEA presentan déficit en la capacidad de prestar atención a los objetos juntamente con otras personas.

Dificultad en el desarrollo de las capacidades de atención conjunta es un ejemplo claro de alteración temprana de las capacidades socio-comunicativas y socio-emocionales en el TEA.

En la alteración de la atención conjunta de iniciativa en el TEA existe menor tendencia a iniciar episodios comunicativos donde se comparte afecto positivo con otra persona.

Las dificultades en la atención conjunta como iniciativa implican fallas en la tendencia a iniciar espontáneamente episodios que tienen como fin compartir experiencias afectivas con otra persona acerca de un objeto o situación en el TEA.

Los niños con TEA realizan muy pocos gestos declarativos.

Las investigaciones sugieren que los niños con autismo en edad preescolar tienen dificultades en la atención conjunta como iniciativa, lo cual está relacionado con diferencias individuales de la alteración social.

Los niños con TEA tienen dificultades para compartir con otras personas de manera espontánea intereses y objetivos comunes, lo que se manifiesta en alteraciones al mostrar y señalar.

EI TEA está constituido por alteraciones en el output social o en la generación flexible de conductas sociales. Un objetivo fundamental de la intervención es el mejorar la capacidad para iniciar de forma espontánea y flexible conductas socio-comunicativas.

Los niños con TEA muestran una grave alteración en el inicio de conductas de atención conjunta para compartir de forma espontánea su propia experiencia con los otros.

En el TEA existen serios problemas con la atención conjunta y con la adopción de perspectivas.

Los niños con TEA muestran una alteración en el desarrollo de la habilidad de seguimiento de la dirección de la mirada y en gestos de señalar de otros.

En el TEA existen alteraciones en el proceso temprano de auto-organización del aprendizaje social lo que genera subsiguientes problemas en el desarrollo conductual.

Los niños con TEA rara vez participan en juegos simbólicos y en la adopción del rol de otra persona.

La educación de los padres es un componente fundamental en la intervención efectiva en niños con TEA.

Los niños con TEA tienen una dificultad en la orientación diádica hacia otras personas, lo que tiene un impacto en la capacidad predictiva de seguimiento de la mirada y en el desarrollo de habilidades simbólicas posteriores.

Señala la hipótesis de una continuidad funcional del desarrollo entre las deficiencias de la atención conjunta y la teoría de la mente en el TEA.

La atención conjunta se constituye en una de las condiciones para el desarrollo comunicativo, emocional y simbólico en el TEA. 


\section{Resultados}

A continuación, se presentan en detalle los hallazgos derivados de la literatura revisada.

\section{La atención conjunta y su papel en el desarrollo de la cognición social}

Las habilidades de atención conjunta hacen referencia a la capacidad que tiene el niño de coordinar su atención con otra persona en relación con un objeto o situación (Mundy \& Thorp, 2005). Estas se constituyen en uno de los indicadores del desarrollo cognitivo, emocional, comunicativo, social y del lenguaje del niño entre los nueve y los doce meses (Mundy, 1995, 2003; Mundy, Kasari \& Sigman, 1992; Mundy, Sigman \& Kasari, 1994) (Ver figura 2). En ese momento, el niño empieza a comprender que al igual que él, las otras personas son agentes intencionales (Tomasello, 1995). Los agentes intencionales tienen metas y hacen elecciones activas de medios culturales con el uso del lenguaje (Tomasello, 2009). La intencionalidad se observa cuando el niño expresa un mensaje a otra persona, toca al adulto, establece contacto visual y gesticula hacia el objeto (Owens, 2001). Esa intención permite compartir intereses comunes y en años posteriores contribuye con el desarrollo del vocabulario, con la regulación de la conducta y con la gestión de las emociones (Adamsony McArthur, 1995; Mundy y Thorp, 2005).

Figura 2. Atención conjunta y su relación con el desarrollo psicológico del niño

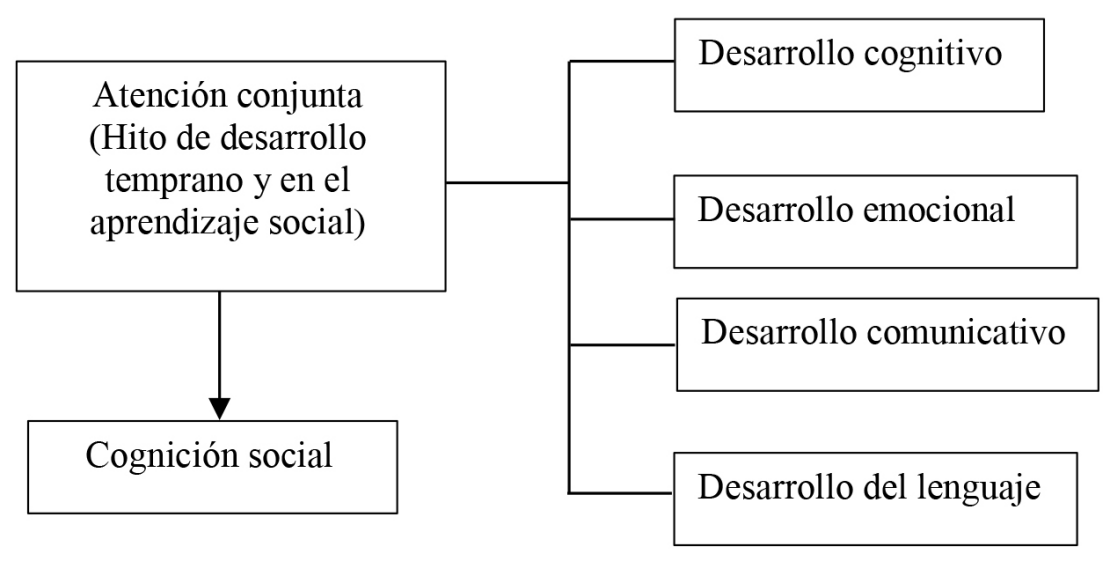

Las habilidades de atención conjunta se constituyen en una fase de humanización debido a que están basadas en su naturaleza compartida con otros (Rivière, 2000). Además, estas habilidades proporcionan las bases en los bebés para comenzar a compartir experiencias con otros y negociar significados compartidos, también proporcionan un contexto para el desarrollo del conocimiento del mundo y sobre los otros (Moore \& Dunham, 1995). Esto quiere decir que el niño aprende a leer múltiples lenguajes, lo que tiene un significado emocional y cultural relacionado con la búsqueda de ese lugar simbólico en el que confluyen infinidad de voces, y en donde cada ser humano comienza a construir sentido (Reyes, 2007).

Para que surjan las habilidades de atención conjunta, es importante la calidad de la comunicación que establece el adulto con el niño. Es por ello que se afirma que el desarrollo infantil se potencia cuando hay una interacción compartida y enriquecida con el adulto (Schertz, Hannah \& Odom, 2007). El adulto debe reconocer las necesidades del niño respondiendo a sus balbuceos o a sus verbalizaciones (Prelock, Calhoun, Morris \& Plat, 2011). Es realmente muy importante atender a sus señales y seguir sus intereses. Por ejemplo, si el niño señala algún objeto que le llama la atención, el adulto debe decir el nombre de ese objeto; si el niño sonríe o balbucea, el adulto puede emplear palabras para expresar aquello que siente que está haciendo sonreír al niño (Schertz, Hannah \& Odom, 2007).
También es necesaria la expresividad y los gestos del adulto al hablar con el bebé, acercándole la cara, arrullándolo, cantándole canciones, escuchando música junto a él, jugando a encontrar un objeto escondido, contando historias, hablando acerca de los objetos que despiertan su interés, etc. (Sussman, 1999; Reyes, 2007; Cabrejo, 2007 Prelock, Calhoun, Morris \& Plat, 2011). El rostro y la voz de los adultos empiezan a movilizar el pensamiento del bebé para construirlo como sujeto social y cultural a través de los diversos intercambios comunicativos (Reyes, 2007). Estas interacciones inician con la atención conjunta la cual hace posible comunicarse con los demás con el uso del lenguaje. De esta manera, se inicia la impresión de huellas de la experiencia humana comprensible a los otros (Vigotsky, 1995a).

La habilidad de interacción comunicativa no verbal que el bebé desarrolla, ayuda a que atraviese con facilidad los momentos de frustración inicial que experimenta al no lograr expresar lo que desea de manera verbal (Sussman, 1999). Esta habilidad no verbal se dirige a los gestos con significado que el bebé realiza en la interacción dialógica con el adulto. Con el desarrollo de estas habilidades, el bebé empieza a aprender quienes son los otros, quién es él, también le permiten conocer cómo funciona el mundo para descifrar sentidos y significados (Cabrejo, 2007).

Las investigaciones muestran que las carencias comunicativas durante los primeros años afectan la calidad del aprendizaje, el desarrollo del pensamiento, el lenguaje y la consolida- 
ción de los vínculos afectivos (Landau, Smith \& Jones, 1988; Huttenlocher et al, 1991). Las investigaciones también señalan que las habilidades de atención conjunta pueden predecir las capacidades de comunicación que el niño tendrá en los siguientes dos años (Tomasello, 2009). Por esta razón, se afirma que la experiencia de la interacción con el bebé desde el comienzo de la vida, ofrece la oportunidad para descifrarse, expresarse, acceder a la cultura y aprender, por ejemplo, acerca de la musicalidad de las palabras (Reyes, 2007). En estas interacciones es importante observar las primeras habilidades de atención conjunta del bebé para tener una idea acerca de su desarrollo comunicativo inicial.

Las habilidades de atención conjunta permiten que dos personas identifiquen un interés común en algún objeto y ambas están conscientes de este foco de atención compartida, lo que posibilita el desarrollo de la interacción comunicativa (Tomasello, 2007). En esa interacción, los adultos ayudan a que los más pequeños comprendan el mundo a través de las acciones con el uso de los objetos en un entorno dialógico para facilitar el ingreso a la cultura. El uso de objetos posibilita transformar el mundo y el propio comportamiento (Vigotsky, 1995a, 1995b).

La atención conjunta implica una respuesta interactiva por parte de la escucha, al coordinar la atención entre un objeto y una persona en un contexto social en el que se comparte una experiencia (Adamson y McArthur, 1995). Su naturaleza es intersubjetiva, lo que significa que algo se comparte y eso es lo que permite la comunicación. La intersubjetividad es tanto el marco de significados como el conocimiento compartido (Tomasello et al, 2005). Cuando el niño y el adulto reconocen la existencia de la intersubjetividad aceptan que existe un propósito de comunicarse (Tomasello, 2009).

Las habilidades de atención conjunta se complejizan. Al inicio implican únicamente la coordinación social de la atención visual como cuando el bebé señala (gesto deíctico) un juguete a un adulto (Mundy y Thorp, 2005). Más adelante, esta habilidad se transforma y se integra con fenómenos psicológicos como compartir ideas, intenciones y emociones con los demás (Tomasello, 1995).

Las habilidades de atención conjunta se refieren a: (1) señalar para compartir la experiencia con los demás y (2) comprender que el otro tiene mente, deseos, necesidades e intenciones. Esas habilidades se adquieren en las interacciones naturales del niño con las personas (Rivière, 1998) y se expresan a través de una mirada larga y fija del bebé hacia el adulto y el objeto, la sonrisa social que expresa emociones de alegría, movimientos de cabeza hacia el objeto, vocalizaciones, levantamiento de las manos y las piernas. Estas formas de expresión en el bebé se constituyen en indicadores que señalan que se está incluyendo en la actividad de comunicación afectivo-emocional con los adultos cercanos (Solovieva y Quintanar, 2012).

Cuando las habilidades de atención conjunta se consolidan desde los primeros años de vida en el niño, más adelante le permiten reflexionar conscientemente sobre el comportamiento, los objetivos y las intenciones de los demás y sobre el comportamiento y la voluntad de sí mismo (Brownell, 2011). Las habilidades de la atención conjunta están afectadas en el TEA (Tager-Flusberg, 1999; Baron-Cohen et al. 1992; Mundy, 2003; Tomasello 1995). Estas alteraciones generan dificultades en el desarrollo de las capacidades socio-comunicativas y socio-emocionales en el niño (Mundy y Sigman,1989) (Ver figura 3). En algunos casos, el niño con TEA no tiene ninguna conducta intencionada de relación con otros acerca de algo mediante signos (Rivière, 2001b), lo que dificulta establecer vínculos emocionales. En otras situaciones, existe dificultad para dividir y alternar la atención del niño entre el objeto y el adulto (Mundy y Thorp, 2005; Mundy, 2017). Los niños con TEA tienen déficit en la duración y en la frecuencia de alternar la mirada entre el adulto y el objeto (Bruinsma, Koegel \& Koegel, 2004). Cuando un niño no advierte que el adulto está intentando cautivar su atención para compartir un objeto o situación, no hay interacción entre los dos (Tomasello, 2009), por lo que se afecta la relación del niño con el mundo y con los otros (Vigotsky, 1997).

Figura 3. Relación TEA y atención conjunta

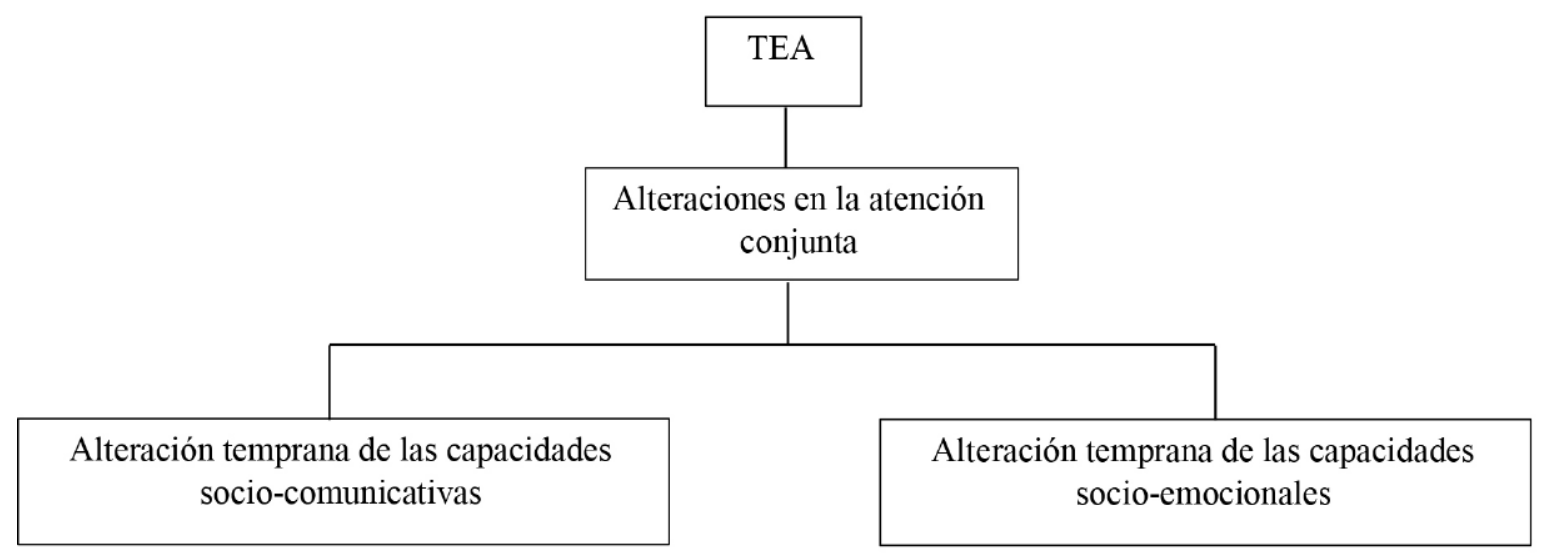




\section{Interacciones diádicas}

A los seis meses los bebés interactúan de manera diádica (bebé-objeto; bebé-otra persona). Esto quiere decir que cuando el bebé se interesa por los objetos, disminuyen los contactos con el adulto y cuando se interesa por el adulto disminuye el interés por los objetos. Cuando el bebé interactúa con el objeto lo explora con sus manos, lo sacude, lo lleva a su boca. Esto muestra que el bebé está interesado en el objeto. La interacción de la diada bebé-otra persona se caracteriza por la bidireccionalidad y la reciprocidad (Tomasello, 2009). De esta forma surge la conversación, y cuando el bebé vocaliza, el adulto responde dando significado a sus expresiones. Esto anima a que el bebé continúe produciendo vocalizaciones porque empieza a identificar que lo que hace consigue la atención del adulto, intercambiándose expresiones faciales y miradas entre ambos.

De acuerdo a investigaciones realizadas por Bruinsma, Koegel \& Koegel (2004), se ha encontrado que en los niños con TEA se altera la interacción de la diada bebé-otra persona. También se han identificado menos acercamientos sociales y más acercamientos a los objetos, y en muchos casos, la relación con los objetos es estereotipada.

\section{Interacciones sociales triádicas}

Entre los nueve y los doce meses el niño comienza a coordinar e integrar sus interacciones con los objetos y las personas (Tomasello, 2009), lo que da como resultado un triángulo amoroso referencial constituido por el niño, el adulto y el objeto (Reyes, 2007). En estas interacciones triádicas se comparten comunicativamente objetos y/o situaciones con los otros. El niño alterna la mirada del objeto al adulto y viceversa (Bruinsma, Koegel \& Koegel, 2004). Cuando el niño y el adulto se interesan por un mismo objeto, el adulto habla del mismo (dice su nombre o sus características). Es así como inicia el juego de la comunicación en el que el niño empieza a participar alternativamente en turnos interactivos, a través de sonrisas, gestos, miradas, pausas y vocalizaciones.

Los niños con TEA tienen dificultades para prestar atención a los objetos y a las personas simultáneamente, lo que genera déficit para adaptarse a la perspectiva perceptual de los demás, es decir, para considerar el punto de vista del otro
(Mundy, 2017). Asimismo, los niños con TEA tienen dificultades para participar en actividades colaborativas, relativas a la intencionalidad compartida, lo que implica: la comprensión de la acción intencional y la motivación de compartir estados psicológicos con los demás (Benassi, s.f.).

\section{La mente dialógica}

Se requiere de la interacción social con el lenguaje con el niño para que él aprenda que el otro tiene mente, que tiene deseos e intenciones. Esto se basa en el supuesto de que los demás son como nosotros (Rivière, 2000), para que advierta que el otro es un sujeto de experiencia y comparta intersubjetivamente la mente con él. En este sentido, la función esencial del lenguaje es compartir y mostrar el mundo mental (Rivière, 2001b). Es así como se inician las interacciones de atención conjunta, la cual se constituye en una característica de la cognición social (Tomasello, 2009). Asimismo, esta última se refiere al conjunto de capacidades que hacen posible a un sujeto la construcción de representaciones mentales de las relaciones entre él y los demás (Baron-Cohen, 1990). En otras palabras, la atención conjunta y la cognición social están estrechamente vinculadas, pues la atención conjunta posibilita el desarrollo de la cognición social (Mundy, 2003; 2017).

En los niños con TEA existen dificultades para compartir y mostrar el mundo mental (Rivière, 2001), lo que hace que se dificulte leer al otro, comprender sus deseos, representar internamente sus estados mentales, establecer interacciones comunicativas, cooperar. Esto tiene como consecuencia que las acciones de otras personas carezcan de sentido y sean difíciles de comprender.

\section{La dedicación conjunta como habilidad de la comproba- ción de la atención}

La dedicación conjunta se refiere a que los niños participan en interacciones sociales mediatizadas por un objeto, lo que quiere decir que comienzan a estar en sintonía con la atención que los adultos dirigen a los objetos y a la conducta que observan de ellos (Tomasello, 2009).

En la figura 4 se presentan los tres tipos principales de interacción atencional de acuerdo con investigaciones de Carpenter, Nagell y Tomasello (1998).

Figura 4. Tipos de interacción atencional

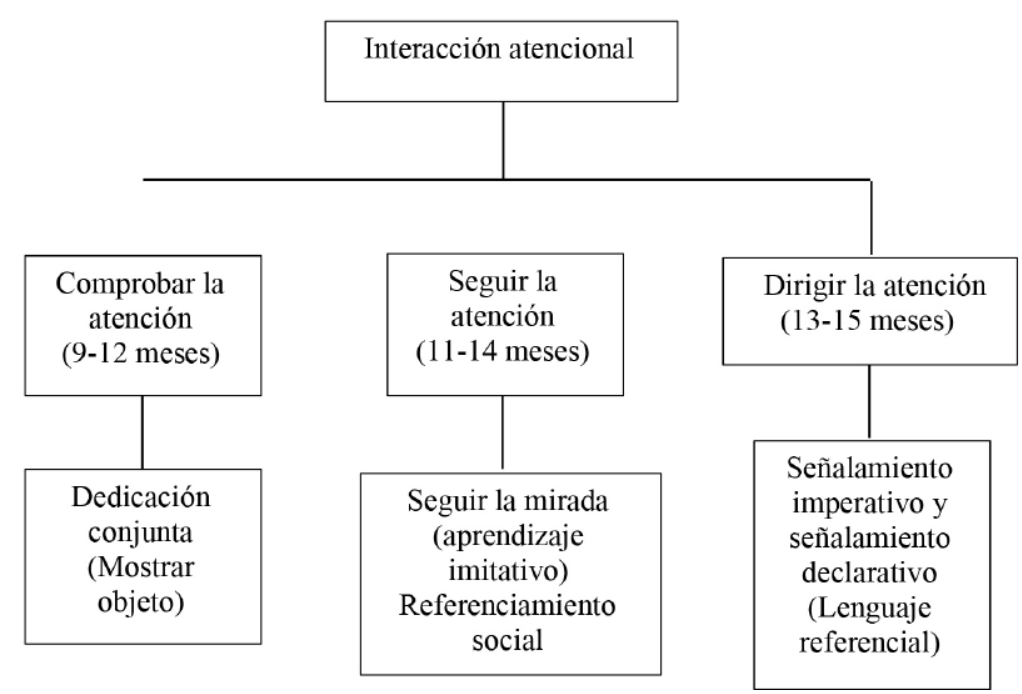


En los niños con TEA se observan dificultades para el desarrollo de la comprobación de la atención del otro. Del mismo modo, en los niños con TEA resulta difícil compartir acciones con los demás; en efecto, no señalan con el dedo algo que les interesa para compartir experiencias y no suelen iniciar las interacciones (Rivière, 1998, 2000, 2001).

\section{Seguimiento de la mirada}

Entre los nueve y los doce meses, los bebés empiezan a mirar lo que los adultos miran, durante periodos de tiempo más largos. Esta es una habilidad que ayuda al establecimiento de vínculos sociales iniciales. Los bebés siguen la mirada hacia el objeto al que se están refiriendo y modifican el foco de atención de los adultos hacia los objetos que son interesantes para ellos (Tomasello, 2009). Asimismo, esta habilidad permite que el adulto enseñe al bebé a identificar señales y a dirigir su atención (Mundy, 2003). Es así como los bebés empiezan a adoptar un punto de vista común con el otro (Mundy, 1995). La práctica de participar en interacciones comunicativas, permite compartir el punto de vista propio con el otro, lo cual es crucial tanto para el desarrollo social como para el aprendizaje social (Mundy, 1995). El bebé expresa su afecto positivo o negativo por algún objeto o situación, llamando la atención del adulto con la mirada para que el adulto realice alguna acción (Mundy, 2003).

Existen dos formas de seguimiento de la mirada. La primera se refiere a que el niño observa un objeto interesante y hace contacto visual con el adulto para compartir experiencias con él. Ese contacto visual ayuda a que el otro vea lo que le interesa al niño. La segunda se refiere a que el adulto observa algún objeto o situación, y llama la atención del niño con gestos y expresiones para compartir con él una experiencia afectiva. Ese tipo de contacto visual hace que el niño vea lo que le interesa al adulto.

En los niños que presentan TEA existe una dificultad en el seguimiento de la mirada y en el contacto visual, no miran a los adultos para comprender situaciones que les interesan, extrañan o sorprenden (Rivière, 2000). El niño tiene problemas para coordinar la atención con la mirada entre el adulto y el objeto. El niño con TEA tiene dificultad para utilizar su contacto visual para iniciar una atención compartida con el adulto y además, para seguir la línea de la interacción del adulto considerando un objeto o situación, lo que genera dificultades en los vínculos sociales y emocionales iniciales y posteriores (Woods \& Wetherby, 2008).

En investigaciones se ha encontrado que los niños con TEA que aprenden a seguir la mirada de otras personas desarrollan el lenguaje verbal (Mundy, 2003, 2017). La capacidad de nombrar objetos es parte de la atención conjunta, la cual permite a los niños con TEA aprender mejor el vocabulario (Mundy, 1995).

\section{Adopción de perspectivas}

La atención conjunta implica la adopción de perspectivas y se caracteriza tanto por la dualidad de los roles (hablar-escuchar), como por la capacidad para comprender que otras personas pueden tener pensamientos y creencias distintos de los nuestros. El adulto señala o mira un objeto y el niño responde, y viceversa. Esto significa que cada uno de los participantes coge el turno para interactuar y sincronizar sus respuestas.

En los niños con TEA se observa una dificultad para adoptar perspectivas porque con frecuencia centran su atención en el objeto. Existe dificultad para participar activamente de la interacción comunicativa con el otro (Escorcia Mora \& Baixauli Fortea, 2012).

\section{Atención conjunta y juego simbólico}

Con la participación del niño en actividades características de su edad de desarrollo psicológico (comunicación afectivo emocional de 0 a 1 año, juego manipulación con objetos de 1 a 3 años), la atención conjunta hace contribuciones al desarrollo del pensamiento simbólico humano. En un primer momento, el niño interactúa con los objetos, lo que facilita la consolidación de sus imágenes internas porque se identifican las propiedades y características esenciales de estos (formas, colores, materiales diversos, tamaños). Después surge el juego simbólico cuando el niño usa objetos como sustitutos de otros y representa acciones con gestos o expresiones faciales. En niños con TEA se presentan alteraciones en la atención conjunta y en el juego simbólico (Kasari, Freeman \& PapareIla, 2006). Sin embargo, en investigaciones se ha encontrado que cuando los niños con TEA mejoran sus habilidades de atención conjunta también mejora el juego simbólico durante la interacción con el adulto, además que los tipos y niveles de juego son más sofisticados (Kasari, Freeman \& Paparella, 2001).

\section{Evaluación de la atención conjunta en el TEA}

Los padres son las personas más cercanas al niño, por esta razón, deben observarlo en sus actividades diarias. Es así como se determina en un primer momento cómo se está desarrollando la atención conjunta, observando cómo se comunica el bebé con el otro, cómo establece conexiones sociales y cómo percibe oportunidades sociales a través del juego (Chang, Shih, Landa, Kaiser \& Kasari, 2018).

A nivel de evaluación clínica, el objetivo es la detección de las alteraciones que se presentan en la atención conjunta tempranamente, considerando los indicadores de la dinámica del desarrollo del niño. Entre estos se puede señalar la posibilidad de realizar acciones con diversos objetos con o sin ayuda del adulto, sustituir un objeto por otro, participar en el juego propuesto por el adulto. Se enfatiza la importancia de la evaluación clínica cualitativa, debido a que puede, de manera decisiva, influir positivamente sobre el desarrollo del niño (Solovieva y Quintanar, 2014). Esta forma de evaluación es construida sobre la base de la zona de desarrollo próximo y la estructura de la actividad. Esto quiere decir que se considera el tipo de apoyo que necesita el niño de parte del adulto, la estructura y el objetivo de la edad psicológica.

En síntesis, considerando las investigaciones revisadas en este artículo, en el cuadro 1 se presentan los indicadores de identificación de riesgo temprano de alteración de la atención conjunta en el TEA. Estos indicadores podrían señalar alguna dificultad que precisa la necesidad de intervención especializada. 
Tabla 2. Indicadores de identificación de riesgo temprano de alteración de la atención conjunta en el TEA

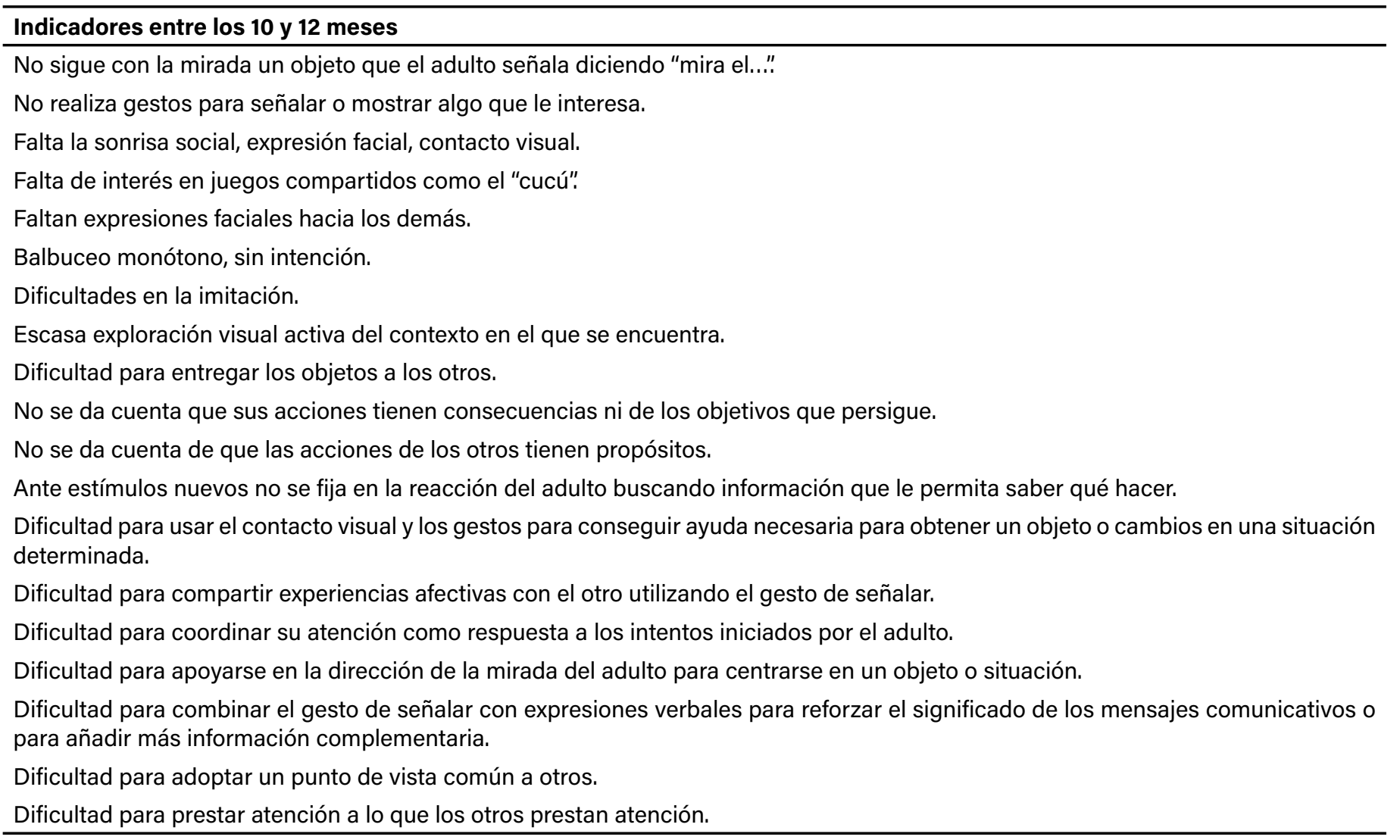

\section{Intervención dirigida al desarrollo de la atención conjunta en el TEA}

En las interacciones de intervención con el niño con TEA, el adulto puede utilizar el juego debido a que esta es la actividad principal en la infancia. El adulto debe ser sensible a las necesidades de desarrollo psicológico del pequeño y de acuerdo a ello utilizar el juego que considere que es apropiado. Por ejemplo, al inicio puede usar el juego con objetos para promover el significado de acciones, objetos y palabras, mientras que el juego simbólico para propiciar la imagen interna de los objetos y la anticipación de acciones (Solovieva y Quintanar, 2012). Más adelante, el adulto podrá utilizar el juego de roles sociales para promover el sentido de la actividad y la colaboración, y el juego narrativo para que el niño pueda poner en palabras sus pensamientos y para que atribuya significado a su experiencia (González-Moreno, 2018a, González-Moreno, 2018b).

El adulto debe asumir una actitud receptiva que le permita estar sincronizado con las necesidades e intereses del niño con TEA para responder con calidez e interés. El adulto debe seguir la dirección del niño para que así se desarrolle la atención conjunta (Kasari et al, 2015). Además, el adulto se debe involucrar observando y dándole información respecto a eso que le interesa al pequeño (Mundy, 2017). Es así como se estimula la capacidad cognitiva de aprender acerca de la atención conjunta (Mundy, 1995, 2003). Asimismo, esto ayuda a que el niño inicie una participación activa en la interacción. Igualmente es necesario ayudar a que el niño sienta que el adulto está con él, por lo que se sugiere estar frente a él física y emocionalmente, imitar lo que él hace, interpretar sus acciones usando palabras sencillas que correspondan a sus acciones, sonidos o gestos. El adulto también puede hacer comentarios respecto a las acciones que inicia el niño para mostrarle el interés respecto a su acción y para darle información respecto a lo que puede aprender respecto a un objeto o situación. De la misma manera, es necesario que el adulto ayude al niño a tomar turnos a través del intercambio de comunicación predecible que le propone al pequeño (Mundy, 2017).

El rol del adulto es facilitar en el niño con TEA la comprensión de su entorno y de las relaciones sociales, provocando su curiosidad, lo que lo va a animar a seguir aprendiendo cosas nuevas, además de generar vínculos afectivos que contribuyen con su desarrollo socio-emocional y socio-comunicativo (Sussman, 1999; Prelock, Calhoun, Morris \& Plat, 2011).

En intervención es necesario aumentar el inicio de iniciativas de atención conjunta, por lo que se debe ayudar a los niños con TEA a mirar a un objeto, después girarse y luego compartir eso que ven con alguien (Kasari, Paparella, Freeman \& Jahromi, 2008; Kasari, Rotheram-Fuller, Locke \& Gulsrud, 2012; Kasari et al, 2015).

Las investigaciones muestran la necesidad de que los profesionales que trabajan con niños con TEA (neuropsicólogos, psicólogos, fonoaudiólogos y pedagogos infantiles), se asocien con las familias para fomentar interacciones con sus niños pequeños. Ellos les enseñan: cómo ayudar al niño a prestar atención, cómo involucrarlo en la comunicación bidireccional, 
cómo ayudarlo a imitar y entender lo que otros dicen y hacen, y le muestran cómo interactuar con otras personas (Sussman, 1999). Además, el diseño de la intervención colaborativa con la familia posibilita que el niño transfiriera habilidades sociales, como por ejemplo, la habilidad de establecer vínculos con otros, la habilidad de colaborar para lograr un objetivo común, la habilidad de expresarse y de escuchar al otro en diversas situaciones de la vida (González-Moreno, 2018b).

\section{Discusión}

Es un desafío comprender las implicaciones que tiene el desarrollo de las habilidades de atención conjunta en el desarrollo infantil del TEA (en la cognición social). Estas permiten la comunicación intencional, el desarrollo de los gestos para compartir la experiencia, y se constituyen, también, en una forma de desciframiento sobre cómo funciona el mundo para el ingreso al mundo de lo simbólico, facilitando un punto de vista común de referencia mental (Mundy, 2003). Además, estas habilidades tienen un papel crucial en el aprendizaje del lenguaje. Por ejemplo, la atención conjunta permite delimitar la zona en la que el niño tiene que buscar al nuevo objeto que corresponde con una nueva palabra (Mundy, 1995, 2017). Eso significa que la atención conjunta ayuda al niño a auto-organizarse para identificar qué palabra va con cada objeto (Mundy, 2003).

De la misma manera, la atención conjunta está relacionada con la teoría de la mente, es decir, con la habilidad para comprender y predecir la conducta de otras personas, sus conocimientos, sus intenciones y sus creencias (Tirapu-Ustárroz et al., 2007). Esta se construye a través de experiencias que posibilitan ser capaz de mirar al mismo objeto con la otra persona y ver que la otra persona responde emocionalmente de una forma similar; en otras palabras, se trata de entender el comportamiento de otros porque el comportamiento sigue a su atención (Mundy, 2003, 2017). Las capacidades de atención conjunta ayudan a construir la capacidad para pensar en los pensamientos de otras personas (Mundy, 2017). En el TEA, uno de los problemas más importantes se refiere a la dificultad de pensar en lo que otros están pensando (Mundy et al., 2016).

Las investigaciones señalan que en los niños con TEA se afecta el compartir la atención con el otro para cooperar, lo que genera dificultades en el desarrollo de las interacciones sociales y en el establecimiento de los vínculos emocionales (Mundy, 2017).

Del mismo modo, las investigaciones en el desarrollo infantil del niño con TEA permiten reflexionar respecto a la necesidad de identificar de manera temprana alteraciones en la atención conjunta, con el objetivo de iniciar la intervención que se requiera y para reducir su impacto negativo en el desarrollo social, comunicativo y emocional.

Se constituye en un reto empezar a desarrollar las habilidades de atención conjunta en los niños que presentan TEA, para que puedan compartir experiencias con otras personas. Con las interacciones que el adulto provoca con el uso del lenguaje y diversos medios de la cultura (objetos, imágenes, cuentos), se puede ayudar a organizar el pensamiento del niño con TEA. Cuanto más se sabe sobre cómo aprenden los niños con TEA, más específico se puede ser en lo que se intenta hacer en las intervenciones tempranas y más impacto se tendrá (Mundy, 2003). El programa de intervención se dirige hacia la formación o el desarrollo de los aspectos débiles de la atención conjunta, para garantizar un efecto sistémico positivo en su desarrollo general, que se refiere al desarrollo de todas las esferas que conforman su vida psíquica: aprendizaje, afectividad, motivos e intereses, cognición, personalidad y comportamiento. Las investigaciones sugieren que la mejora en el inicio de la atención conjunta en el niño con TEA predice su desarrollo cognitivo, emocional, comunicativo y del lenguaje posterior (Mundy et al., 2016). Asimismo, predice la mejora en los síntomas del TEA (Kasari, Paparella, Freeman \& Jahromi, 2008; Mundy, 2017). Por ejemplo, si el niño mejora en atención conjunta aprende más palabras (Chiu et al., 2008).

Cuando el niño con TEA desarrolla habilidades de atención conjunta, logra percibir emociones propias y de los otros con mayor facilidad, entiende lo que piensan los demás en determinadas situaciones, evalúa mentalmente las interacciones con los otros y participa de manera más activa en el contexto social y cultural (Mundy, Sigman \& Kasari, 1994; Mundy, 1995, 2017).

Diversos especialistas intervienen cuando no aparecen las habilidades de atención conjunta en el niño: neuropsicólogos, psicólogos, fonoaudiólogos y pedagogos infantiles. Estos especialistas deben integrar sus acciones a través de la realización de actividades interprofesionales. También es necesario involucrar de manera activa a los padres del niño para que comprendan formas claras de interacción con sus pequeños con TEA; ellos tienen más oportunidades para trabajar de manera más eficaz la atención conjunta desde la casa, el ambiente natural (Mundy, 2003).

Para el desarrollo de la atención conjunta se requiere proponer acciones interesantes y relevantes que tengan un uso social y que sean accesibles para el niño; de esta manera, él se va a sentir involucrado emocionalmente.

La identificación temprana de las señales que muestran alteraciones en la atención conjunta es clave para el éxito en el desarrollo psicológico del niño que presenta TEA. Es así como se pueden proponer estrategias de intervención que consideren las necesidades específicas del niño en su desarrollo psicológico, contribuyendo con un mejor pronóstico y por consiguiente, con una mejor calidad de vida para el pequeño y para su familia. Se concluye que la atención conjunta se debe trabajar en la intervención temprana en niños con TEA (Mundy, 2003). 


\section{Referencias}

Adamson, L. y McArthur, D. (1995). Joint attention, affect and culture. En C. Moore y P. Dunham (Eds.), Joint attention: Its origins and role in development. Hillsdale, New Jersey: Lawrence Erlbaum. 205-222.

Barbaro, J. \& Dissanayake, C. (2009). Autism spectrum disorders in infancy and toddlerhood: a review of the evidence on early signs, early identification tools, and early diagnosis. J Dev Behav Pediatr, 30(5):447-59.

Baron-Cohen, S. (1988). Social and pragmatic deficits in autism: Cognitive or affective? Journal of Autism and Developmental Disorders, 18: 379-401.

Baron Cohen, S., Jolliffe, T., Mortimore, C. \& Robertson, M. (1997). Another advanced Test of Theory of Mind: Evidence from Very High Functioning Adults with Autism and Asperger Syndrome. J. Child Psychol, 38(7).

Baron-Cohen, S (1990). Autismo: un trastorno cognitivo específico de "ceguera de la mente". International Review of Psychiatry, 2, 81-90.

Baron-Cohen, S., Allen, J., \& Gillberg, C. (1992). Can autism be detected at 18 months? The needle, the haystack, and the CHAT. British Journal of Psychiatry, 161, 839-843.

Baron-Cohen, S. (1995). The eye direction detector (EDD) and the shared attention mechanism (SAM): Two cases for evolutionary psychology. In Moore, C. \& Dunham, P.J. (Eds), Joint Attention: Its Origins and Role in Development. Lawrence Erlbaum Associates, Hillsdale, 41-59.

Benassi, J. (s.f). Investigaciones con bebés. Desarrollo simbólico e indicadores tempranos de autismo. UNL. Santa Fe. Argentina. Texto Nº: Compartir intenciones. Estudios comparados con bebés y chimpancés.

Brownell, C. (2011). Early Developments in Joint Action. Rev Philos Psychol, 2(2), 193-211. doi: 10.1007/s13164011-0056-1Bruinsma, Y., Koegel, R.L. \& Koegel, L.K. (2004). Joint Attention and Children with Autism: A Review of the Literature. Mental Retardation and Developmental Disabilities Research Reviews. 10, 169-175.

Cabrejo, E. (2007). Lenguaje y construcción de la representación del otro en los niños y las niñas. En: Lenguaje y saberes infantiles. Flor Alba Santamaría V. Bogota, Cátedra UNESCO en desarrollo del niño.

Carpenter, M., Nagell, K. \& Tomasello, M. (1998). Social cognition, join attention and communicative competence from 9 to 15 months of age. Monographs of the society for research in child development 63.

Chiu, P.H., Kayali, M.A., Kishida, K.T., Tomlin, D., Klinger, L.G., Klinger, M.R. \& Montague, P.R. (2008). Self-responses along cingulate cortex reveal quantitative neural phenotype for high-functioning autism. Neuron, 57, 463-473.

Corbetta, M., Patel, G. \& Shulman, G.L. (2008). The reorienting system of the human brain: from environment to theory of mind. Neuron, 58, 306-324.

Chang, Y.C., Shih, W., Landa, R., Kaiser, A. \& Kasari, C. (2018). Symbolic Play in School-Aged Minimally Verbal Children with Autism Spectrum Disorder. J Autism
Dev Disord, 48(5). 1436-1445. doi: 10.1007/s10803017-3388-6.

Elkonin, D. B. (1980). Psicología del juego. Madrid: Visor Libros.

Escorcia Mora, C. \& Baixauli Fortea, I. (2012). Comunicación, atención conjunta e imitación en el trastorno del espectro autista. International Journal of Developmental and Educational Psychology, 3 (1), 49-57.

González-Moreno, C.X. (2018a). Intervención en un niño con autismo mediante el juego. Rev. Fac. Med, 66(3):365-74. Spanish. doi: http://dx.doi. org/10.15446/revfacmed.v66n3.62355.

González-Moreno, C.X. (2018b). El juego como estrategia para el desarrollo del lenguaje en un niño con Trastorno del Espectro Autista desde el ámbito de la educación inclusiva. IE Revista de Investigación Educativa de la Rediech, 9(17), 9-31.

Huttenlocher, J., Haight, W., Bryk, A., Seltzer, M., \& Lyons, T. (1991). Early vocabulary growth: relation to language input and gender. Developmental Psychology, 27, 236-248.

Kasari, C., Sigman, M., Mundy, P. \& Yirmiya, N. (1990). Affective sharing in the context of joint attention interactions of normal, autistic, and mentally retarded children. J. Autism Dev. Disord., 20, 87-100.

Kasari, C., Freeman, S., \& Paparella, T. (2001). Early intervention in autism: Joint attention and symbolic play. International Review of Research in Mental Retardation, 23, 207-237.

Kasari, C., Freeman, S. \& Paparella, T. (2006). Joint attention and symbolic play in young children with autism: a randomized controlled intervention study. Journal of Child Psychology and Psychiatry, 47(6), 611-620 doi:10.1111/j.1469-7610.2005.01567.x

Kasari, C., Paparella, T., Freeman, S. \& Jahromi, L.B. (2008), Language outcome in autism: randomized comparison of joint attention and play interventions. Journal of Consulting and Clinical Psychology, 76(1), 125-137.Kasari, C., Rotheram-Fuller, E., Locke, J. \& Gulsrud, A. (2012). Making the connection: randomized controlled trial of social skills at school for children with autism spectrum disorders. J. Child Psychol. Psyc., 53, 431-439.

Kasari, C., Gulsrud, A., Paparella, T., Hellemann, G. \& Berry, K. (2015). Randomized comparative efficacy study of parent-mediated interventions for toddlers with autism. Journal of Consulting and Clinical Psychology, 83(3), 554-563.

Kennedy, D.P. \& Adolphs, R. (2012). The social brain in psychiatric and neurological disorders. Trends in Cognitive Sciences, 16(11), 559-572. https://doi. org/10.1016/j.tics.2012.09.006

Landau, B., Smith, L. \& Jones, S. (1988). The importance of shape in early lexical learning. Cognitive Development, 3(3), 299-321. https://doi.org/10.1016/08852014(88)90014-7

Moore, C. \& Dunham, P.J. (1995). Joint Attention: Its Origins and Role in Development. Hillsdale, NJ, US: Lawrence Erlbaum Associates, Inc.

Mundy, P., Kasari, C. \& Sigman, M. (1992). Nonverbal communication, affective sharing, and intersubjectivity. Infant Behavior and Development, 15(3), 377-381. https://doi.org/10.1016/0163-6383(92)80006-G 
Mundy, P., Sigman, M., \& Kasari, C. (1994). Joint attention, develop-mental level, and symptom presentation in children with autism. Development and Psychopathology, 6(3), 389-401. https://doi.org/10.1017/ S0954579400006003

Mundy, P. (1995). Joint attention and social-emotional approach behavior in children with autism. Development and Psychopathology. 7(1), 63-82. https:// doi.org/10.1017/S0954579400006349

Mundy, P. (2003). The neural basis of social impairments in autism: The role of the dorsal medial-frontal cortex and anterior cingulate system. Journal of Child Psychology and Psychiatry. 44, 793-809.

Mundy, P. y Thorp, D. (2005). Atención conjunta y autismo: Teoría, evaluación y neurodesarrollo. En: J. Matos, P. González, M. Llorente y C. Nieto. (Comp.). Nuevos desafíos en autism: el futuro es hoy. Madrid: APNA.

Mundy, P., Kim, K., McIntyre, N., Lerro, L., \& Jarrold, W. (2016). Brief report: Joint attention and information processing in children with higher functioning autism spectrum disorders. Journal of Autism and Developmental Disorders, 1-6, doi:10.1007/ s10803-0162785-6.

Mundy, P. (2017). A review of joint attention and social-cognitive brain systems in typical development and autism spectrum disorder. European Journal of Neuroscience, 1-18, doi:10.1111/ejn.13720

Owens, R. (2001). Desarrollo del lenguaje. España: Pearson. Prentice Hall.

Prelock, P.; Calhoun, J.; Morris, H. \& Plat, G. (2011). Supporting parents to facilitate communication and joint attention in their young children with autism spectrum disorders: two pilot studies. Topics in Language Disorders. 31(3), 210-234. doi: 10.1097/TLD. Ob013e318227bd3f

Reyes, Y. (2007). La casa imaginaria. Bogotá: Editorial Norma. Rivière, A. (1997). Tratamiento y definición del espectro autista I. Relaciones sociales y comunicación. En Á. Rivière y J. Martos (Coords.), El tratamiento del autismo. Nuevas perspectivas. Madrid: Instituto de Migraciones y Servicios Sociales.

Rivière, A. (1998). El tratamiento del autismo como trastorno del desarrollo: principios generales. En A. Rivière y J. Martos (Comp.). El tratamiento del autismo. Nuevas perspectivas. Madrid: APNA-IMSERSO.

Rivière, A. (2000). ¿Cómo aparece el autismo? Diagnóstico temprano e indicadores precoces del trastorno autista. En A. Rivière y J. Martos (Comp.) El niño pequeño con autismo. Madrid: APNA.

Riviére, A. y Martos, J. (2000). El niño pequeño con autismo. Madrid: Asociación de Padres de Niños Autistas.

Rivière, Á. (2001a). Autismo, orientaciones para la intervención educativa. Editorial Trotta.

Rivière, A. (2001b). Lenguaje y autismo. Conferencia en Buenos Aires (1999). En Valdez, D. (2001). Autismo, enfoques actuales para padres y profesionales de la salud y la educación. 2 Tomos. Buenos Aires. Fundec.

Scaife, M. \& Bruner, J. (1975) The capacity for joint visual attention in the infant. Nature, International Journal of science. 253, 265-266.

Schertz, Hannah H, \& Odom, S. L. (2007). Promoting joint attention in toddlers with autism: a parentmediated developmental model. Journal of autism and developmental disorders, 37(8), 1562-75. doi:10.1007/ s10803-006-0290-z

Solovieva, Y., Machinskaya, R., Quintanar-Rojas, L., Bonilla, R. y Pelayo, H. (2009). Neuropsicología y electrofisiología del TDA en la edad preescolar. México: Universidad Autónoma de Puebla.

Solovieva, Y. y Quintanar, L. (2012). La actividad de juego en la edad preescolar. México: Trillas.

Solovieva, Y. y Quintanar, L. (2014). Evaluación del desarrollo para niños preescolares menores. México: Benemérita Universidad Autónoma de Puebla.

Sussman, F. (1999). More than words: Helping parents promote communication and social skills in children with autism spectrum disorders. Toronto, Ontario, Canada: A. Hanen Centre.

Tager-Flusberg, H. (1999). A Psychological Approach to Understanding the Social and Language Impairments in Autism. Int Rev Psychiatry. 11(4), 325-334. doi: 10.1080/09540269974203

Tirapu-Ustárroz, J., Pérez-Sayes, G., Erekatxo-Bilbao, M. \& Pelegrín-Valero, C. (2007). ¿Qué es la teoría de la mente? Rev Neuro. 44 (8), 479-489.

Tomasello, M. (1995). Joint attention as social cognition. En Join attention its origins and role in development (ED C, MOORE y P. DUNHAM). P. 103-130. Hillsdale, NJ: Lawrence Erlbaum.

Tomasello, M., Carpenter, M., Call, J., Behne, T., \& Moll, H. (2005). Understanding and sharing intentions: The origins of cultural cognition. Behavioral and Brain Sciences, 28, 675-691.

Tomasello, M. (2009). Los orígenes culturales de la cognición humana. Buenos Aires: Amorrortu.

Vigotsky, L.S. (1995a). Obras escogidas. Tomo III. Madrid: Visor.

Vigotsky, L.S. (1995b). Pensamiento y lenguaje. Buenos Aires: Ediciones Fausto.

Vigotsky, L.S. (1997). Obras escogidas. Tomo V. Madrid: Visor. Wimmer, H. \& Perner, J. (1983). Beliefs about beliefs: representation and constraining function of wrong beliefs in young children's understanding of deception. Cognition, 13(1), 103-128. https://doi.org/10.1016/00100277(83)90004-5

Wolf, I., Dziobek, I. \& Heekeren, H.R. (2010). Neural correlates of social cognition in naturalistic settings: a model-free analysis approach. Neurolmage, 49(1), 894-904. https://doi.org/10.1016/j.neuroimage.2009.08.060

Woods, J. J., \& Wetherby, A. M. (2008). Early identification of and intervention for infants and toddlers who are at risk for autism spectrum disorder. Language, Speech, and Hearing Services in Schools, 34, 180193. doi:10.1044/0161-1461

Zwaigenbaum, L., Bryson, S. \& Garon, N. (2013). Early identification of autism spectrum disorders. Behavioural Brain Research. 251(1):133-146. https://doi.or$\mathrm{g} / 10.1016 / \mathrm{j} . \mathrm{bbr} .2013 .04 .004$ 